\title{
Rabbits killing birds revisited
}

\author{
Jimin Zhang ${ }^{\text {a }}$, Meng Fan ${ }^{\mathrm{a}, *, 1}$, Yang Kuang ${ }^{\mathrm{b}, 2}$ \\ ${ }^{a}$ School of Mathematics and Statistics, and Key Laboratory for Vegetation Ecology, \\ Northeast Normal University, 5268 Renmin Street, Changchun, Jilin 130024, PR China \\ ${ }^{\mathrm{b}}$ Department of Mathematics and Statistics, Arizona State University, Tempe, AZ 85287-1804, United States
}

Received 27 July 2005; received in revised form 14 December 2005; accepted 12 January 2006

Available online 10 March 2006

\begin{abstract}
We formulate and study a three-species population model consisting of an endemic prey (bird), an alien prey (rabbit) and an alien predator (cat). Our model overcomes several model construction problems in existing models. Moreover, our model generates richer, more reasonable and realistic dynamics. We explore the possible control strategies to save or restore the bird by controlling or eliminating the rabbit or the cat when the bird is endangered. We confirm the existence of the hyperpredation phenomenon, which is a big potential threat to most endemic prey. Specifically, we show that, in an endemic prey-alien prey-alien predator system, eradication of introduced predators such as the cat alone is not always the best solution to protect endemic insular prey since predator control may fail to protect the indigenous prey when the control of the introduced prey is not carried out simultaneously.

(c) 2006 Elsevier Inc. All rights reserved.
\end{abstract}

MSC: 92D25

Keywords: Bird conservation; Control strategies; Hyperpredation process; Apparent competition; Mathematical models

\footnotetext{
* Corresponding author.

E-mail addresses: mfan@nenu.edu.cn (M. Fan), kuang@asu.edu (Y. Kuang).

${ }^{1}$ Supported by the National Natural Science Foundation of PR China (No. 10201005), the Key Project on Science and Technology of the Education Ministry of PR China.

${ }^{2}$ Partially supported by NSF grants DMS-0077790 and DMS-0342388.
} 


\section{Introduction}

It is known that species that consume different resources and that do not interact directly can still influence each other's population growth rates if they share common natural enemies such as predators, parasites, or pathogens. There is evidence that alternate abundances between species may occur under a broad ranges of indirect population interactions, which are widely thought to have important consequences for community structures. One of the important types of indirect interaction is the so-called apparent competition [9], where two host species that do not compete directly have adverse effects on one another through a shared natural enemy. This occurs when an increase in the abundance of one species results in a decrease in the numbers of another species solely from the consequence of the natural enemy becoming more abundant because it has an alternative prey species.

Recently, models of apparent competition have linked exotic predators to both native and exotic prey, suggesting that the introduction of a novel prey species can indirectly cause the extinction of indigenous prey [4,6]. This form of apparent competition, also termed hyperpredation, occurs when an indigenous prey species suffers an increase in predation pressure from an exotic predator that is sustained by an exotic prey [19]. In [4,6], Courchamp et al. discussed the effect of rabbit overgrazing on island ecosystems. Successful alien grazers are generally able to eat a large variety of native plants, and the result is frequently a dramatic impoverishment of the quantity and quality of indigenous flora. They also reported that, on Hawaiian Islands chain, rabbits alone were responsible for eliminating 26 species of plants between 1903 and 1923, and are believed to be responsible for the decline or extinction of several reptile and bird species.

Overgrazing by rabbits can cause many direct and indirect effects on indigenous vertebrate species especially on seabirds. For example, they compete with seabirds for existing burrows and reduce the plant cover for terrestrial nesting seabirds, affecting seabird reproductive success. However, the effects of rabbits on indigenous vertebrate species can be very complex, especially when introduced cats are also present.

Cats are opportunistic predators, eating what is easily available. They switch prey according to their relative availability. When the rabbits and birds have equal availability, the cats prefer to prey on bird species, which are more easily caught, due to the fact that the indigenous prey species (bird) has inferior anti-predator characteristic compare to the introduced prey species such as rabbits $[4,6]$. It is also known that in many islands, introduced mammals such as rabbits, rats and mice are the main prey of cats in winter. These introduced mammals not only allow cats to subsist and maintain during the absence of bird in winter but also enable the cat population to increase significantly. In other words, these introduced mammals lead to increased predation pressure on the bird species. The combination of a low adaptation to predation and induced high predation pressure due to an introduced mammal can lead to dramatic indigenous prey population decrease, up to the point of total extinction.

The harm caused by the introduced predators on an oceanic islands is widely known, and control programs are largely recognized as the best way to restore ecosystems. In many cases, introduced predators (cats) and prey (rabbits) are both present. The effects of cats on indigenous species are direct, while no direct effect of the rabbits on the indigenous prey were often observed and no correlated change seemed to be involved. That is to say, the most obvious effects of rabbits 
are often indirect. Therefore, priority is then generally given to the control of cats and, in some situations, control of rabbits may not be considered at all. However, when rabbits and cats are both present, the hyperpredation process can lead to the extinction of some indigenous prey species and increase the difficulty of control of cats.

Although more general apparent competition has been studied theoretically $[9,1]$, the hyperpredation process has received relatively little attention in conservation biology. Courchamp et al. [6] established an interesting mathematical model modelling the interactions among an indigenous prey (bird), an introduced prey (rabbit) and a common predator (cat) in an island setting where the direct effects of rabbits on birds are neglected. Through this model, the existence of hyperpredation process is demonstrated in theory. In their subsequent research [4] (published earlier than [6]), they constructed another mathematical model coupling the population dynamics of such three species and discussed the control strategies for protecting the birds.

Their model is governed by the following system of ordinary differential equations

$$
\begin{aligned}
& \frac{\mathrm{d} B}{\mathrm{~d} t}=r_{\mathrm{b}} B\left(1-\frac{B}{K_{\mathrm{b}}}\right)-\eta_{\mathrm{b}} B R-\frac{\alpha B}{\alpha B+R} \mu_{\mathrm{b}} C, \\
& \frac{\mathrm{d} R}{\mathrm{~d} t}=r_{\mathrm{r}} R\left(1-\frac{R}{K_{\mathrm{r}}}\right)-\frac{R}{\alpha B+R} \mu_{\mathrm{r}} C-\lambda_{\mathrm{r}} R, \\
& \frac{\mathrm{d} C}{\mathrm{~d} t}=r_{\mathrm{c}} C\left(1-\frac{\mu_{\mathrm{b}} \mu_{\mathrm{r}} C}{\mu_{\mathrm{r}} B+\mu_{\mathrm{b}} R}\right)-\lambda_{\mathrm{c}} C,
\end{aligned}
$$

where

- $B(t), R(t)$ and $C(t)$ denote the number of individuals at time $t$ in the bird, rabbit and cat, respectively.

- $r_{\mathrm{b}}, r_{\mathrm{r}}$ and $r_{\mathrm{c}}$ are the intrinsic growth rates of the bird, rabbit and cat, respectively.

- $\mu_{\mathrm{b}}$ and $\mu_{\mathrm{r}}$ are the predation rates of the cat on the bird and the rabbit, respectively.

- $K_{\mathrm{b}}$ and $K_{\mathrm{r}}$ are the carrying capacities for the bird and the rabbit, respectively.

- $\alpha$ is the preference of the cat, i.e., the bird/rabbit ratio in the diet of cats.

- $\eta_{\mathrm{b}}$ represents the per capita reduction of bird birth rate due to rabbit's direct and indirect competition. Throughout [4], it has been set to 0 to account for cases where the rabbit has no direct effect on the bird.

- $\lambda_{\mathrm{r}}$ and $\lambda_{\mathrm{c}}$ represent the control effort for controlling the rabbits and the cats, respectively.

This theoretical study indicates that the control of the introduced prey (the rabbit) facilitates the control of the introduced predator (the cat). Predator control may fail to protect the indigenous prey if control of the introduced prey is not undertaken simultaneously.

Although the conclusions are plausible, the model formulation of (1.1) is problematic. The modelling approach of (1.1) is similar to that in [5] for modelling the middle predator release effect. The model in [5] also has a few model formulation problems (see [8]). For example, if the bird is absent in the three-species system, then (1.1) reduces to 


$$
\begin{aligned}
& \frac{\mathrm{d} R}{\mathrm{~d} t}=r_{\mathrm{r}} R\left(1-\frac{R}{K_{\mathrm{r}}}\right)-\underline{\mu_{\mathrm{r}} C}-\lambda_{\mathrm{r}} R, \\
& \frac{\mathrm{d} C}{\mathrm{~d} t}=r_{\mathrm{c}} C\left(1-\frac{\mu_{\mathrm{r}} C}{R}\right)-\lambda_{\mathrm{c}} C,
\end{aligned}
$$

where the predation of the cat on the rabbit only depends on the size of the cat while it has nothing to do with the rabbit here. This is simply not realistic. This situation also occurs in the case when the rabbit is absent in (1.1).

Another serious drawback of (1.1) is that it fails to model the interference between predator individuals, which has been shown to exist by a large number of field and laboratory experiments and observations [16]. One key component of a predator-prey relationship is the predator's rate of feeding upon prey, namely the predator's functional responses. Various mechanistic derivations show $[2,3,7]$ that the functional responses are often predator-dependent. Jost and Ellner [13] carried out systematically statistical studies and obtained significant evidence of predator dependence in the functional response. In (1.1), the cat's functional responses are only prey-dependent, which fails to account for the interference between the predators.

Although the model (1.1) is investigated in [4], and the possible occurrence of the hyperpredation in (1.1) is mentioned, it failed to present any explicit criteria for it to take place. Courchamp et al. [4] also discussed some control strategies to protect the birds. Again, their study is neither complete nor systematic. The principal aim of this paper is to develop a more plausible model and revisit the dynamics of indigenous prey-alien prey-alien predator interactions. We are also interested in formulating sound control strategies to protect or restore endangered endemic preys.

In the next Section 2, we derive the cat's functional response via the classical Holling time budget argument and formulate an indigenous prey-introduced alien prey-introduced alien predator model. We then investigate the basic dynamics of this model. We demonstrate in theory the existence of the apparent competition (hyperpredation) phenomenon. We then systematically investigate several control strategies to protect or to restore the endangered native prey species. In the discussion section, we examine additional biological implications of our mathematical findings and state some biologically motivated mathematical questions for future study. Throughout this paper, extensive computational results are presented to illustrate or complement our mathematical findings.

\section{Model construction}

The principal aim of this section is to establish a mathematical model, which overcomes the model formulation problems of (1.1). First, we derive suitable candidates for the cat's functional responses based on the classical Holling time budget argument. The details are basically the same as those in deriving the functional responses for the rats and the cats in [8] where the authors' motivation is studying the mechanisms generating meso-predator release behavior. For convenience, we provide the details below. 


\subsection{Cat's functional response}

In the following, $B(t), R(t)$ and $C(t)$ denote the population density at time $t$ for bird, rabbit and cat, respectively.

We assume that the total time spent by a cat for gathering food from both bird and rabbit is $T$, which can be divided into four parts:

- $T_{\mathrm{cs}}$ : the time spent by cat for searching bird and rabbit. We assume the cat's searching time for bird or for rabbit is same, since cat is an opportunist and will attack the one that is first available.

- $T_{\text {chb }}$ : the time spent by a cat for handling caught birds.

- $T_{\text {chr }}$ : the time spent by a cat for handling a caught rabbits.

- $T_{\mathrm{cw}}$ : the time wasted by cat for interfering with other cats.

We define

- $\alpha_{\mathrm{cb}}$ as the capture rate of cat for bird and

- $\alpha_{\text {cr }}$ as the capture rate of cat for rabbit.

Hence the differences in the ability for birds and rabbits to be captured is adequately taken into account in the capture rates, $\alpha_{\mathrm{cb}}$ and $\alpha_{\mathrm{cr}}$.

The number of bird(rabbit) caught per cat is proportional to the bird (rabbit) density $B(R)$ and the search time. Hence

- the total number of bird caught per cat $N_{\mathrm{cb}}=\alpha_{\mathrm{cb}} B T_{\mathrm{cs}}$ and

- the total number of rabbit caught per cat $N_{\mathrm{cr}}=\alpha_{\mathrm{cr}} R T_{\mathrm{cs}}$.

For simplicity, we assume that the total time spent for handling caught bird (rabbit) is equal to the product of the total number of caught bird (rabbit) and the expected handling time $t_{\mathrm{chb}}\left(t_{\mathrm{chr}}\right)$ per unit of bird (rabbit). That is,

$$
T_{\mathrm{chb}}=\alpha_{\mathrm{cb}} t_{\mathrm{chb}} B T_{\mathrm{cs}}, \quad T_{\mathrm{chr}}=\alpha_{\mathrm{cr}} t_{\mathrm{chr}} R T_{\mathrm{cs}} .
$$

Cats are solitary predators. The wasted time $T_{\mathrm{cw}}$ due to interference is given by the product of the number of encounters between cats $N_{\mathrm{cc}}$ and the time wasted per encounter $t_{\mathrm{cw}}$. If cats move randomly, $N_{\text {cc }}$ can be expressed as

$$
N_{\mathrm{cc}}=\alpha_{\mathrm{cc}} T_{\mathrm{cs}}(C-1),
$$

where $\alpha_{\mathrm{cc}}$ is the rate of encounter between cats, related to both their speed of movement and the range at which they sense each other. The total time wasted due to encounters between cats is then given by

$$
T_{\mathrm{cw}}=\alpha_{\mathrm{cc}} T_{\mathrm{cs}}(C-1) t_{\mathrm{cw}}
$$

Hence,

$$
T=T_{\mathrm{cs}}+T_{\mathrm{chb}}+T_{\mathrm{chr}}+T_{\mathrm{cw}}=\left[1+\alpha_{\mathrm{cb}} t_{\mathrm{chb}} B+\alpha_{\mathrm{cr}} t_{\mathrm{chr}} R+\alpha_{\mathrm{cc}} t_{\mathrm{cw}}(C-1)\right] T_{\mathrm{cs}} .
$$


Consequently, the cat's functional responses are

$$
\frac{N_{\mathrm{cb}}}{T}=\frac{\alpha_{\mathrm{cb}} B}{1+\alpha_{\mathrm{cb}} t_{\mathrm{chb}} B+\alpha_{\mathrm{cr}} t_{\mathrm{chr}} R+\alpha_{\mathrm{cc}} t_{\mathrm{cw}}(C-1)}
$$

and

$$
\frac{N_{\mathrm{cr}}}{T}=\frac{\alpha_{\mathrm{cr}} R}{1+\alpha_{\mathrm{cb}} t_{\mathrm{chb}} B+\alpha_{\mathrm{cr}} t_{\mathrm{chr}} R+\alpha_{\mathrm{cc}} t_{\mathrm{cw}}(C-1)} .
$$

These functions are called Beddington-DeAngelis functional responses. Complete analytical results on Beddington-DeAngelis functional response predator-prey model can be found in $[3,11,12]$.

\subsection{The model}

Now we are ready to establish the predator-prey system modelling the interaction among the bird, the rabbit and the cat. Let $x(t), y(t), z(t)$ be the densities of the bird, the rabbit and the cat, respectively. In the absence of the cat, we assume that both the bird and the rabbit grow logistically

$$
\dot{x}=r_{1} x\left(1-\frac{x}{K_{1}}\right), \quad \dot{y}=r_{2} y\left(1-\frac{y}{K_{2}}\right) .
$$

Here $r_{1}, r_{2}$ are the intrinsic growth rates of the bird and the rabbit and $K_{1}, K_{2}$ are the environmental carrying capacity of the bird and the rabbit, respectively. In the absence of the bird and the rabbit, the cats decline exponentially, i.e., $\dot{z}=-\mathrm{d} z$, where $d$ is the cat's death rate. Now we assume that the bird, the rabbit and the cat live together. The cat preys on both the bird and the rabbit according to the functional response derived above. Let $e_{1}$ and $e_{2}$ be the conversion rates of the bird and the rabbit into the biomass of the cat. Since rabbits can have negative effect on the growth of the birds via direct and indirect competitions, we account for this by $\eta x y$ (like the term $\eta \mathrm{BR}$ in (1.1)). Here $\eta_{\mathrm{b}}$ represents the per capita reduction of bird birth rate due to rabbit's direct and indirect competition. We thus obtain the following model:

$$
\begin{aligned}
& \dot{x}=r_{1} x\left(1-\frac{x}{K_{1}}\right)-\eta x y-\frac{\alpha_{\mathrm{cb}} x z}{F(x, y, z)}, \\
& \dot{y}=r_{2} y\left(1-\frac{y}{K_{2}}\right)-\frac{\alpha_{\mathrm{cr}} y z}{F(x, y, z)}, \\
& \dot{z}=-d z+\frac{e_{1} \alpha_{\mathrm{cb}} x z+e_{2} \alpha_{\mathrm{cr} y z}}{F(x, y, z)},
\end{aligned}
$$

where

$$
F(x, y, z)=1+\alpha_{\mathrm{cb}} t_{\mathrm{chb}} x+\alpha_{\mathrm{cr}} t_{\mathrm{chr}} y+\alpha_{\mathrm{cc}} t_{\mathrm{cw}}(z-1) .
$$


For simplicity, we re-scale the parameters by the following substitutions

$$
\begin{array}{lll}
a_{1}=\frac{\alpha_{\mathrm{cb}}}{1-\alpha_{\mathrm{cc}} t_{\mathrm{cw}}}, & a_{2}=\frac{\alpha_{\mathrm{cr}}}{1-\alpha_{\mathrm{cc}} t_{\mathrm{cw}}}, & b_{1}=\frac{e_{1} \alpha_{\mathrm{cb}}}{1-\alpha_{\mathrm{cc}} t_{\mathrm{cw}}},
\end{array}
$$

The model on substituting into (2.1) becomes

$$
\begin{aligned}
& \dot{x}=r_{1} x\left(1-\frac{x}{K_{1}}\right)-\eta x y-\frac{a_{1} x z}{1+\alpha x+\beta y+\gamma z}, \\
& \dot{y}=r_{2} y\left(1-\frac{y}{K_{2}}\right)-\frac{a_{2} y z}{1+\alpha x+\beta y+\gamma z}, \\
& \dot{z}=-d z+\frac{b_{1} x z+b_{2} y z}{1+\alpha x+\beta y+\gamma z} .
\end{aligned}
$$

The harm caused by introduced species on oceanic islands is widely known, and control programs are largely recognized as the best way to restore ecosystems. This paper has two main objectives. First, we will demonstrate in theory the existence of the hyperpredation phenomenon. Then we will systematically study the control strategies to protect the birds by controlling the rabbits and/or the cats. As in [4], we apply a control effort $u_{\mathrm{r}}$ on the rabbit population and a control effort $u_{\mathrm{c}}$ on the cat population. This yields the following controlled bird-rabbit-cat system:

$$
\begin{aligned}
& \dot{x}=r_{1} x\left(1-\frac{x}{K_{1}}\right)-\eta x y-\frac{a_{1} x z}{1+\alpha x+\beta y+\gamma z}, \\
& \dot{y}=r_{2} y\left(1-\frac{y}{K_{2}}\right)-\frac{a_{2} y z}{1+\alpha x+\beta y+\gamma z}-u_{\mathrm{r}} y, \\
& \dot{z}=-d z+\frac{b_{1} x z+b_{2} y z}{1+\alpha x+\beta y+\gamma z}-u_{\mathrm{c}} z .
\end{aligned}
$$

Due to the biological significance of (2.3), we will deal with the solutions of (2.3) with positive initial values, i.e., $x(0)>0, y(0)>0, z(0)>0$. We also assume that all the parameters in (2.2) are positive unless explicitly stated otherwise. From standard mathematical arguments, one can easily claim that for any $x(0)>0, y(0)>0, z(0)>0$, Eq. (2.3) has a unique positive solution defined for all $t \geqslant 0$.

\section{Equilibria and their local stability}

In this section, we explore the existence and local stability of boundary and positive equilibria. The possible equilibria or steady states of (2.3) are listed below:

$$
E_{0}:(0,0,0), \quad E_{\mathrm{b}}:\left(K_{1}, 0,0\right), \quad E_{\mathrm{r}}:\left(0, \frac{K_{2}\left(r_{2}-u_{\mathrm{r}}\right)}{r_{2}}, 0\right),
$$


$E_{\mathrm{br}}:\left(x_{4}^{*}, y_{4}^{*}, 0\right)$, where $x_{4}^{*}$ and $y_{4}^{*}$ solve

$$
r_{1}\left(1-\frac{x}{K_{1}}\right)-\eta y=0, \quad r_{2}\left(1-\frac{y}{K_{2}}\right)-u_{\mathrm{r}}=0
$$

$E_{\mathrm{rc}}:\left(0, y_{5}^{*}, z_{5}^{*}\right)$, where $y_{5}^{*}$ and $z_{5}^{*}$ solve

$$
r_{2}\left(1-\frac{y}{K_{2}}\right)-u_{\mathrm{r}}-\frac{a_{2} z}{1+\beta y+\gamma z}=0, \quad-\left(d+u_{\mathrm{c}}\right)+\frac{b_{2} y}{1+\beta y+\gamma z}=0 ;
$$

$E_{\mathrm{bc}}:\left(x_{6}^{*}, 0, z_{6}^{*}\right)$, where $x_{6}^{*}$ and $z_{6}^{*}$ solve

$$
r_{1}\left(1-\frac{x}{K_{1}}\right)-\frac{a_{1} z}{1+\alpha x+\gamma z}=0, \quad-\left(d+u_{\mathrm{c}}\right)+\frac{b_{1} x}{1+\alpha x+\gamma z}=0 ;
$$

$E_{\mathrm{brc}}:\left(x_{7}^{*}, y_{7}^{*}, z_{7}^{*}\right)$, where $x_{7}^{*}, y_{7}^{*}, z_{7}^{*}$ solve

$$
\begin{aligned}
& r_{1}\left(1-\frac{x}{K_{1}}\right)-\eta y-\frac{a_{1} z}{1+\alpha x+\beta y+\gamma z}=0, \\
& r_{2}\left(1-\frac{y}{K_{2}}\right)-u_{\mathrm{r}}-\frac{a_{2} z}{1+\alpha x+\beta y+\gamma z}=0, \\
& -\left(d+u_{\mathrm{c}}\right)+\frac{b_{1} x+b_{2} y}{1+\alpha x+\beta y+\gamma z}=0 .
\end{aligned}
$$

In order to determine the local stability of these equilibria, we first calculate the variational matrix of (2.3). After some straightforward algebraic calculations, we obtain

$$
J(x, y, z)=\left(\begin{array}{lll}
a_{11} & a_{12} & a_{13} \\
a_{21} & a_{22} & a_{23} \\
a_{31} & a_{32} & a_{33}
\end{array}\right),
$$

where

$$
\begin{aligned}
& a_{11}=r_{1}\left(1-\frac{2 x}{K_{1}}\right)-\eta y-\frac{a_{1} z(1+\beta y+\gamma z)}{(1+\alpha x+\beta y+\gamma z)^{2}}, \quad a_{12}=-\eta x+\frac{a_{1} \beta x z}{(1+\alpha x+\beta y+\gamma z)^{2}}, \\
& a_{13}=-\frac{a_{1} x(1+\alpha x+\beta y)}{(1+\alpha x+\beta y+\gamma z)^{2}}, \quad a_{21}=\frac{a_{2} \alpha y z}{(1+\alpha x+\beta y+\gamma z)^{2}}, \\
& a_{22}=r_{2}-u_{\mathrm{r}}-\frac{2 r_{2} y}{K_{2}}-\frac{a_{2} z(1+\alpha x+\gamma z)}{(1+\alpha x+\beta y+\gamma z)^{2}}, \quad a_{23}=-\frac{a_{2} y(1+\alpha x+\beta y)}{(1+\alpha x+\beta y+\gamma z)^{2}}, \\
& a_{31}=\frac{b_{1} z(1+\beta y+\gamma z)-b_{2} \alpha y z}{(1+\alpha x+\beta y+\gamma z)^{2}}, \quad a_{32}=\frac{b_{2} z(1+\alpha x+\gamma z)-b_{1} \beta x z}{(1+\alpha x+\beta y+\gamma z)^{2}}, \\
& a_{33}=-\left(d+u_{\mathrm{c}}\right)+\frac{\left(b_{1} x+b_{2} y\right)(1+\alpha x+\beta y)}{(1+\alpha x+\beta y+\gamma z)^{2}} .
\end{aligned}
$$


The local stability of the equilibrium $\left(x^{*}, y^{*}, z^{*}\right)$ is determined by the eigenvalues of the matrix $J\left(x^{*}, y^{*}, z^{*}\right)$. For $E_{0}$, we have

$$
J\left(E_{0}\right)=J(0,0,0)=\left(\begin{array}{ccc}
r_{1} & 0 & 0 \\
0 & r_{2}-u_{\mathrm{r}} & 0 \\
0 & 0 & -\left(d+u_{\mathrm{c}}\right)
\end{array}\right) .
$$

So we can conclude that

Theorem 3.1. $E_{0}$ is always a saddle node and there cannot be total extinction of the system (2.3) for positive initial conditions.

For $E_{\mathrm{b}}$ and $E_{\mathrm{r}}$, we have

$$
J\left(E_{\mathrm{b}}\right)=\left(\begin{array}{ccc}
-r_{1} & -\eta K_{1} & -\frac{a_{1} K_{1}}{1+\alpha K_{1}} \\
0 & r_{2}-u_{\mathrm{r}} & 0 \\
0 & 0 & -\left(d+u_{\mathrm{c}}\right)+\frac{b_{1} K_{1}}{1+\alpha K_{1}}
\end{array}\right)
$$

and

$$
J\left(E_{\mathrm{r}}\right)=\left(\begin{array}{ccc}
r_{1}-\frac{\eta K_{2}\left(r_{2}-u_{\mathrm{r}}\right)}{r_{2}} & 0 & 0 \\
0 & -\left(r_{2}-u_{\mathrm{r}}\right) & -\frac{a_{2} K_{2}\left(r_{2}-u_{\mathrm{r}}\right)}{r_{2}+\beta K_{2}\left(r_{2}-u_{\mathrm{r}}\right)} \\
0 & 0 & -\left(d+u_{\mathrm{c}}\right)+\frac{b_{2} K_{2}\left(r_{2}-u_{\mathrm{r}}\right)}{r_{2}+\beta K_{2}\left(r_{2}-u_{\mathrm{r}}\right)}
\end{array}\right) .
$$

We can easily reach the following claim.

Theorem 3.2. $E_{\mathrm{b}}=\left(K_{1}, 0,0\right)$ always exists and is stable if

$$
u_{\mathrm{r}}>r_{2}, \quad d+u_{\mathrm{c}}>\frac{b_{1} K_{1}}{1+\alpha K_{1}} .
$$

$E_{\mathrm{r}}$ exists if $r_{2}>u_{\mathrm{r}}$ and it is stable if

$$
\eta>\frac{r_{1}}{K_{2}}, \quad 0 \leqslant u_{\mathrm{r}}<r_{2}\left(1-\frac{r_{1}}{\eta K_{2}}\right), \quad d+u_{\mathrm{c}}>\frac{b_{2} K_{2}\left(r_{2}-u_{\mathrm{r}}\right)}{r_{2}+\beta K_{2}\left(r_{2}-u_{\mathrm{r}}\right)} .
$$

If $0 \leqslant \eta<\frac{r_{1}}{K_{2}}$, then $E_{\mathrm{r}}$ is a saddle point.

Theorem 3.3. $E_{\mathrm{br}}$ exists if

$$
0 \leqslant \eta<\frac{r_{1}}{K_{2}}, 0 \leqslant u_{\mathrm{r}}<r_{2} \quad \text { or } \quad \eta>\frac{r_{1}}{K_{2}}, \quad r_{2}\left(1-\frac{r_{1}}{\eta K_{2}}\right)<u_{\mathrm{r}}<r_{2} .
$$

Moreover, if

$$
d+u_{\mathrm{c}}>\frac{r_{1} r_{2} b_{1} K_{1}+K_{2}\left(r_{2}-u_{\mathrm{r}}\right)\left(r_{1} b_{2}-b_{1} \eta K_{1}\right)}{r_{1} r_{2}\left(1+\alpha K_{1}\right)+K_{2}\left(r_{2}-u_{\mathrm{r}}\right)\left(r_{1} \beta-\alpha \eta K_{1}\right)}
$$

then $E_{\mathrm{br}}$ is stable. Otherwise, $E_{\mathrm{br}}$ is unstable. 
Proof. From (3.1), it follows that

$$
x_{4}^{*}=\frac{K_{1}\left(r_{1} r_{2}-\eta K_{2}\left(r_{2}-u_{\mathrm{r}}\right)\right)}{r_{1} r_{2}}, \quad y_{4}^{*}=\frac{K_{2}\left(r_{2}-u_{\mathrm{r}}\right)}{r_{2}} .
$$

The first half of the theorem (existence part of $E_{\mathrm{br}}$ ) is strightforward. Note that since $x_{4}^{*}$ and $y_{4}^{*}$ solve (3.1), we have

$$
J\left(E_{\mathrm{br}}\right)=\left(\begin{array}{ccc}
-\frac{x_{4}^{*}}{K_{1}} & -\eta y_{4}^{*} & -\frac{a_{1} x_{4}^{*}}{1+\alpha x_{4}^{*}+\beta y_{4}^{*}} \\
0 & -\frac{r_{2}}{K_{2}} y_{4}^{*} & -\frac{a_{2} y_{4}^{*}}{1+\alpha x_{4}^{*}+\beta y_{4}^{*}} \\
0 & 0 & -\left(d+u_{\mathrm{c}}\right)+\frac{b_{1} x_{4}^{*}+b_{2} y_{4}^{*}}{1+\alpha x_{4}^{*}+\beta y_{4}^{*}}
\end{array}\right) .
$$

The proof of the second part of the theorem is now obvious.

The following corollary is simple to use and is straightforward from the above theorem.

Corollary 3.1. $E_{\mathrm{br}}$ exists if

$$
\eta=0, r_{2}>u_{\mathrm{r}} \quad \text { or } \quad r_{2}\left(1-\frac{r_{1}}{\eta K_{2}}\right)<u_{\mathrm{r}}<r_{2} .
$$

Moreover, if

$$
d+u_{\mathrm{c}}>\max \left\{\frac{b_{1}}{\alpha}, \frac{b_{2}}{\beta}\right\},
$$

then $E_{\mathrm{br}}$ is stable.

Theorem 3.4. Assume that

$$
0 \leqslant u_{\mathrm{r}}<r_{2}, \quad 0<d+u_{\mathrm{c}}<\frac{b_{2} K_{2}\left(r_{2}-u_{\mathrm{r}}\right)}{K_{2} \beta\left(r_{2}-u_{\mathrm{r}}\right)+r_{2}},
$$

then $E_{\mathrm{rc}}=\left(0, y_{5}^{*}, z_{5}^{*}\right)$ exists, where

$$
\frac{d+u_{\mathrm{c}}}{b_{2}-\beta\left(d+u_{\mathrm{c}}\right)}<y_{5}^{*}<\frac{\left(r_{2}-u_{\mathrm{r}}\right) K_{2}}{r_{2}} .
$$

Moreover, if

$$
b_{2} \gamma>a_{2} \beta, \quad 0 \leqslant u_{\mathrm{r}}<r_{2}-\frac{r_{1} a_{2}}{a_{1}}, \quad \eta \geqslant \frac{a_{1} r_{2}}{a_{2} K_{2}}
$$

or

$$
\begin{aligned}
& b_{2} \gamma>a_{2} \beta, \quad a_{1}>r_{1} \gamma, \quad 0 \leqslant u_{\mathrm{r}}<r_{2}-\frac{r_{1} a_{2}}{a_{1}}, \quad 0 \leqslant \eta<\frac{a_{1} r_{2}}{a_{2} K_{2}} \quad \text { and } \\
& 0<d+u_{\mathrm{c}}<\frac{b_{2} K_{2}\left[a_{1}\left(r_{2}-u_{\mathrm{r}}\right)-r_{1} a_{2}\right]\left[\eta K_{2} \gamma\left(r_{2}-u_{\mathrm{r}}\right)+\left(a_{1} r_{2}-a_{2} \eta K_{2}\right)-r_{1} r_{2} \gamma\right]}{\left(a_{1} r_{2}-a_{2} \eta K_{2}\right)\left[\left(a_{1} r_{2}-a_{2} \eta K_{2}\right)+K_{2} \beta\left(a_{1}\left(r_{2}-u_{\mathrm{r}}\right)-a_{2} r_{1}\right)\right]},
\end{aligned}
$$

then $E_{\mathrm{rc}}$ is locally asymptotically stable. 
Proof. First we show the existence of $E_{\mathrm{rc}}$. Since $y_{5}^{*}$ and $z_{5}^{*}$ solve (3.2), it is not difficult to see that $\left(y_{5}^{*}, z_{5}^{*}\right)$ is the intersection of the curves

$$
z=-\frac{b_{2} r_{2}}{a_{2} K_{2}\left(d+u_{\mathrm{c}}\right)} y\left[y-\frac{\left(r_{2}-u_{\mathrm{r}}\right) K_{2}}{r_{2}}\right], \quad z=\frac{b_{2}-\left(d+u_{\mathrm{c}}\right) \beta}{\left(d+u_{\mathrm{c}}\right) \gamma} y-\frac{1}{\gamma} .
$$

Consider the function

$$
F(y)=-\frac{b_{2} r_{2}}{a_{2} K_{2}\left(d+u_{\mathrm{c}}\right)} y\left[y-\frac{\left(r_{2}-u_{\mathrm{r}}\right) K_{2}}{r_{2}}\right]-\frac{b_{2}-\left(d+u_{\mathrm{c}}\right) \beta}{\left(d+u_{\mathrm{c}}\right) \gamma} y+\frac{1}{\gamma} .
$$

We see that

$$
F\left(\left(d+u_{\mathrm{c}}\right) /\left(b_{2}-\beta\left(d+u_{\mathrm{c}}\right)\right)\right)>0, \quad F\left(\left(r_{2}-u_{\mathrm{r}}\right) K_{2} / r_{2}\right)<0 .
$$

From the Mean-Value theorem of continuous function, it follows that there exists a

$$
y_{5}^{*} \in\left(\frac{d+u_{\mathrm{c}}}{b_{2}-\beta\left(d+u_{\mathrm{c}}\right)}, \frac{\left(r_{2}-u_{\mathrm{r}}\right) K_{2}}{r_{2}}\right)
$$

such that $F\left(y_{5}^{*}\right)=0 . y_{5}^{*}$ is clearly unique. In addition,

$$
z_{5}^{*}=\frac{b_{2}-\left(d+u_{\mathrm{c}}\right) \beta}{\left(d+u_{\mathrm{c}}\right) \gamma} y_{5}^{*}-\frac{1}{\gamma}>0 .
$$

Therefore, $E_{\mathrm{rc}}$ exists uniquely.

The Jacobian matrix at $E_{\mathrm{rc}}$ takes the form of

$$
J\left(E_{\mathrm{rc}}\right)=J\left(0, y_{5}^{*}, z_{5}^{*}\right)=\left(\begin{array}{ccc}
a_{11} & 0 & 0 \\
a_{21} & a_{22} & a_{23} \\
a_{31} & a_{32} & a_{33}
\end{array}\right),
$$

where

$$
\begin{array}{lc}
a_{11}=r_{1}-\frac{a_{1}}{a_{2}}\left(r_{2}-u_{r}\right)-\left(\eta-\frac{a_{1} r_{2}}{a_{2} K_{2}}\right) y_{5}^{*}, & a_{21}=\frac{a_{2} \alpha y_{5}^{*} z_{5}^{*}}{\left(1+\beta y_{5}^{*}+\gamma z_{5}^{*}\right)^{2}}, \\
a_{22}=-\frac{r_{2} y_{5}^{*}}{K_{2}}+\frac{a_{2} \beta y_{5}^{*} z_{5}^{*}}{\left(1+\beta y_{5}^{*}+\gamma z_{5}^{*}\right)^{2}}, & a_{23}=-\frac{a_{2} y_{5}^{*}\left(1+\beta y_{5}^{*}\right)}{\left(1+\beta y_{5}^{*}+\gamma z_{5}^{*}\right)^{2}}, \\
a_{31}=\frac{b_{1} z_{5}^{*}\left(1+\beta y_{5}^{*}+\gamma z_{5}^{*}\right)-b_{2} \alpha y_{5}^{*} z_{5}^{*}}{\left(1+\beta y_{5}^{*}+\gamma z_{5}^{*}\right)^{2}}, & a_{32}=\frac{b_{2} z_{5}^{*}\left(1+\gamma z_{5}^{*}\right)}{\left(1+\beta y_{5}^{*}+\gamma z_{5}^{*}\right)^{2}}, \\
a_{33}=-\frac{b_{2} \gamma y_{5}^{*} z_{5}^{*}}{\left(1+\beta y_{5}^{*}+\gamma z_{5}^{*}\right)^{2}} . &
\end{array}
$$

If $b_{2} \gamma>a_{2} \beta$, then 


$$
\begin{aligned}
& a_{22}+a_{33}=-\frac{r_{2} y_{5}^{*}}{K_{2}}-\frac{\left(b_{2} \gamma-a_{2} \beta\right) y_{5}^{*} z_{5}^{*}}{\left(1+\beta y_{5}^{*}+\gamma z_{5}^{*}\right)^{2}}<0, \\
& a_{22} a_{33}-a_{32} a_{23}=\frac{r_{2} b_{2} \gamma\left(y_{5}^{*}\right)^{2} z_{5}^{*}}{K_{2}\left(1+\beta y_{5}^{*}+\gamma z_{5}^{*}\right)^{2}}+\frac{a_{2} b_{2} y_{5}^{*} z_{5}^{*}}{\left(1+\beta y_{5}^{*}+\gamma z_{5}^{*}\right)^{3}}>0 .
\end{aligned}
$$

If (3.5) is satisfied, then $a_{11}<0$. If (3.6) is valid, from (3.2), one can solve $y_{5}^{*}$ explicitly although it is somewhat tedious. After substituting $y_{5}^{*}$ into $a_{11}$ and simplifying $a_{11}$, we reach the conclusion that $a_{11}<0$. To conclude, if (3.5) or (3.6) is satisfied, then we have

$$
a_{11}<0, \quad a_{22}+a_{33}<0 \text { and } a_{22} a_{33}-a_{32} a_{23}>0,
$$

which implies that all the three eigenvalues of the characteristic equation of $J\left(E_{\mathrm{rc}}\right)$ have negative real parts. Therefore, $E_{\mathrm{rc}}$ is locally asymptotically stabile.

By carrying out similar arguments as above for $E_{\mathrm{rc}}$, we can obtain that

\section{Theorem 3.5. If}

$$
0<d+u_{\mathrm{c}}<\frac{b_{1} K_{1}}{K_{1} \alpha+1}
$$

then $E_{\mathrm{bc}}=\left(x_{6}^{*}, 0, z_{6}^{*}\right)$ exists, where

$$
\frac{d+u_{\mathrm{c}}}{b_{1}-\alpha\left(d+u_{\mathrm{c}}\right)}<x_{6}^{*}<K_{1} .
$$

Moreover, if

$$
b_{1} \gamma>a_{1} \alpha, \quad u_{\mathrm{r}}>\max \left\{r_{2}-\frac{a_{2}}{\gamma}, r_{2}-\frac{r_{1} a_{2}}{a_{1}}\right\}
$$

and

$$
0<d+u_{\mathrm{c}}<\frac{b_{1} K_{1}\left[r_{1} a_{2}-a_{1}\left(r_{2}-u_{\mathrm{r}}\right)\right]\left[a_{2}-\left(r_{2}-u_{\mathrm{r}}\right) \gamma\right]}{a_{2}\left[K_{1} \alpha\left(r_{1} a_{2}-a_{1}\left(r_{2}-u_{\mathrm{r}}\right)\right)+r_{1} a_{2}\right]},
$$

then $E_{\mathrm{bc}}$ is locally asymptotically stable.

Mathematically, the total extinction of the three populations will never occur since $E_{0}$ is unstable. However, this may not true ecologically since organisms are discrete and can be completely eliminated when the densities become very small. This can happen even though the equilibrium at the origin $E_{0}$ is unstable. Still, several biologically interesting outcomes may arise from this system. The extinction of both the rabbit and the cat populations with the bird population reaching its carrying capacity $\left(E_{\mathrm{b}}\right)$, and the extinction of both the bird and the cat populations with the rabbit reaching its carrying capacity $\left(E_{\mathrm{r}}\right)$ are both possible outcomes of our model system (with different set of parameters). Three other possibilities involve the disappearance of the birds only 

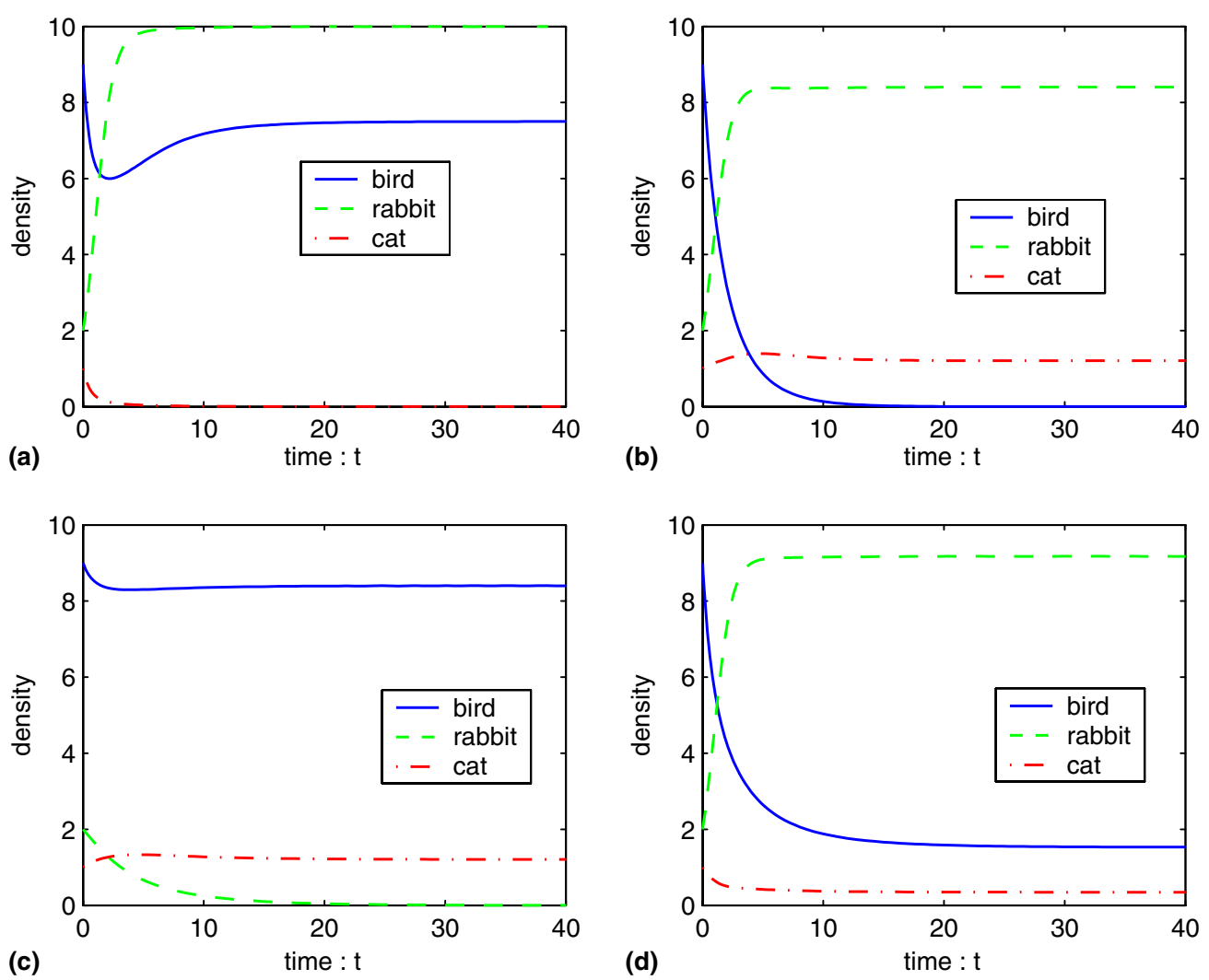

Fig. 1. Here $K_{1}=K_{2}=10$ and $u_{\mathrm{r}}=0, u_{\mathrm{c}}=0, \eta=0.02$. (a) $E_{\mathrm{br}}$ is an attractor $\left(r_{1}=0.8, r_{2}=1.5, a_{1}=4, a_{2}=1, b_{1}=0.4\right.$, $\left.b_{2}=0.3, \alpha=0.2, \beta=0.05, \gamma=3, d=2.2\right)$. (b) $E_{\mathrm{rc}}$ is an attractor $\left(r_{1}=0.8, r_{2}=1.5, a_{1}=4, a_{2}=1, b_{1}=0.4, b_{2}=0.3\right.$, $\alpha=0.2, \beta=0.05, \gamma=3, d=0.5)$. (c) $E_{\mathrm{bc}}$ is an attractor $\left(r_{1}=1.5, r_{2}=0.8, a_{1}=1, a_{2}=4, b_{1}=0.3, b_{2}=0.4, \alpha=0.05\right.$, $\beta=0.2, \gamma=3, d=0.5)$. (d) $E_{\mathrm{brc}}$ is an equilibrium $\left(r_{1}=0.8, r_{2}=1.5, a_{1}=4, a_{2}=1, b_{1}=0.4, b_{2}=0.3, \alpha=0.2, \beta=0.05\right.$, $\gamma=3, d=1.2$.

$E_{\mathrm{rc}}$, or the rabbits only $E_{\mathrm{bc}}$, or the cats only $E_{\mathrm{br}}$. Numerical simulations show that the bird, rabbit and cat can coexist together at an equilibrium $E_{\text {brc }}$ (see Fig. 1(d)), whose expressions are too complex to be presented here. The stability criteria for $E_{\text {brc }}$ are also too complex to be presented here analytically. However, the deterministic nature of the model allows convenient and convincing numerical study.

\section{Apparent competition: rabbit induced bird extinction}

In this section, we will demonstrated theoretically the existence of apparent competition in model (2.2). To this end, we explore the pure indirect effect of rabbit on bird by letting $\eta$ to be zero. That is to say, the interaction among bird, rabbit and cat is governed by the following bird-rabbit-cat model: 


$$
\begin{aligned}
& \dot{x}=r_{1} x\left(1-\frac{x}{K_{1}}\right)-\frac{a_{1} x z}{1+\alpha x+\beta y+\gamma z}, \\
& \dot{y}=r_{2} y\left(1-\frac{y}{K_{2}}\right)-\frac{a_{2} y z}{1+\alpha x+\beta y+\gamma z}, \\
& \dot{z}=-d z+\frac{b_{1} x z+b_{2} y z}{1+\alpha x+\beta y+\gamma z} .
\end{aligned}
$$

Based on the analysis in Section 3, for system (4.1), we can claim that $E_{0}, E_{\mathrm{b}}$ and $E_{\mathrm{r}}$ are unstable. If

$$
\begin{aligned}
& b_{2} \gamma>a_{2} \beta, \quad a_{1} r_{2}>r_{1} a_{2}, \quad a_{1}>r_{1} \gamma, \\
& 0<d<\frac{b_{2} K_{2}\left(a_{1} r_{2}-r_{1} a_{2}\right)\left(a_{1}-r_{1} \gamma\right)}{a_{1}\left(a_{1} r_{2}+K_{2} \beta\left(a_{1} r_{2}-r_{1} a_{2}\right)\right)},
\end{aligned}
$$

then $E_{\mathrm{rc}}$ is stable, while if the last inequality in (4.2) is replaced by

$$
d>\frac{b_{1} K_{1}+b_{2} K_{2}}{1+\alpha K_{2}+\beta K_{2}}
$$

then $E_{\mathrm{br}}$ is stable. When (4.2) is satisfied, the cat has a relatively high growth rate. Therefore, the cat exerts high predation on the bird and the extinction of the bird is not surprising. The bird is in a very dangerous situation. In this case, people usually carry out some control strategy of the cat to protect the bird. Such control strategy is effective if the control effort is appropriately large. When the control effort $u_{\mathrm{c}}$ is greater than $\frac{b_{1} K_{1}+b_{2} K_{2}}{1+\alpha K_{2}+\beta K_{2}}-d$, then $E_{\mathrm{br}}$ is an attractor and the birdrabbit-cat system achieves a new balance at $E_{\mathrm{br}}$. The bird can be successfully saved.

One of our main objectives is to assess the effect of the rabbit on the bird. The countless indirect ecological effects of introduced rabbits on birds can be assessed by studying the changes occurring to ecosystems, following their decline or complete removal, by eradication programmes $[17,18]$. The simplest way to study this quantitatively is to compare the value of the bird at equilibrium under different conditions.

In the following, we will control the rabbit by $u_{\mathrm{r}}$. We achieve this by inserting an additional term $-u_{\mathrm{r}} y$ into the second equation of (4.1). If the control is insufficient (for example, $\left.u_{\mathrm{r}}<r_{2}-r_{1} a_{2} / a_{1}\right)$, then $E_{\mathrm{rc}}$ is still an attractor, and the bird is doomed. However, although such a control is insufficient to protect the bird, it decreases the cat's equilibrium population level (see Fig. 2(b) and (f)). If we increase the control effort $u_{\mathrm{r}}$ further (for example, $u_{\mathrm{r}}>r_{2}-r_{1} a_{2} / a_{1}$ ), then $E_{\mathrm{rc}}$ becomes unstable, and $E_{\mathrm{brc}}$ or $E_{\mathrm{bc}}$ may be the attractor. The bird population can be protected when the control is sufficient (see Fig. 2(c)-(f)). One can also observe that the equilibrium level of the cat population decreases with increasing control effort of the rabbit. To conclude, to control the rabbit is beneficial to the bird. Although the rabbit does not have any direct negative effect on the bird in above discussion, it positively affects the growth of the cat and indirectly asserts heavy predation on the bird through the cat. This mechanism has been termed apparent competition or hyperpredation. The model confirms the detrimental effect of the rabbits' presence to birds, even in the absence of any direct effects.

In the rest of this section, in order to better understand the hyperpredation phenomenon, we will investigate how some parameters in (4.1) affect the dynamics of the bird-rabbit-cat system. 
(a) $u_{r}=0$

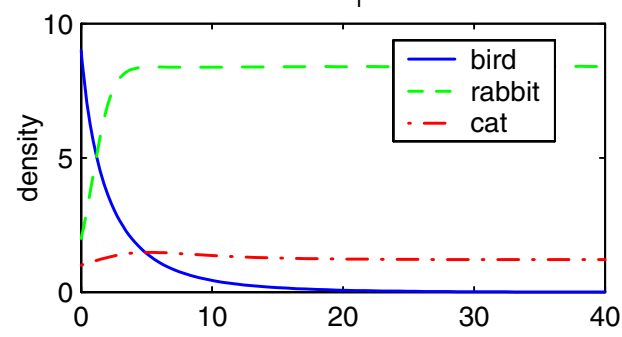

(c) $u_{r}=0.8$

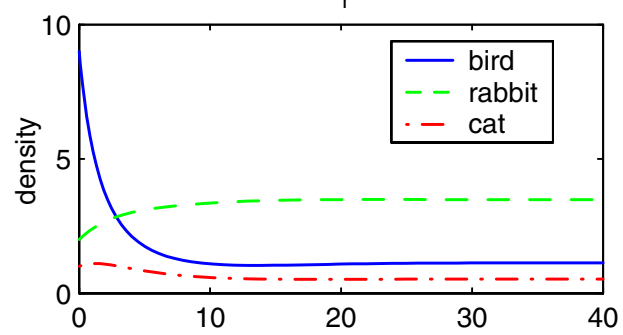

(e) $\mathrm{u}_{\mathrm{r}}=1.7$

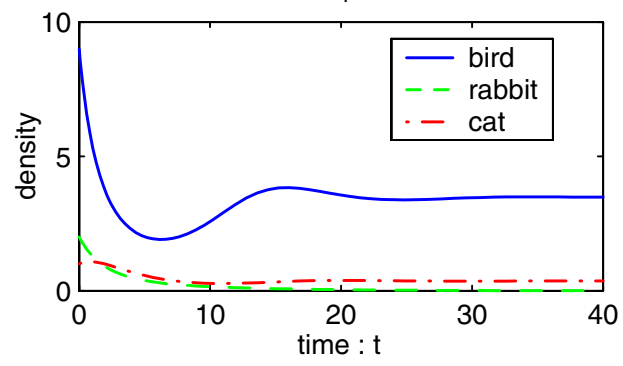

(b) $\mathrm{u}_{\mathrm{r}}=0.4$

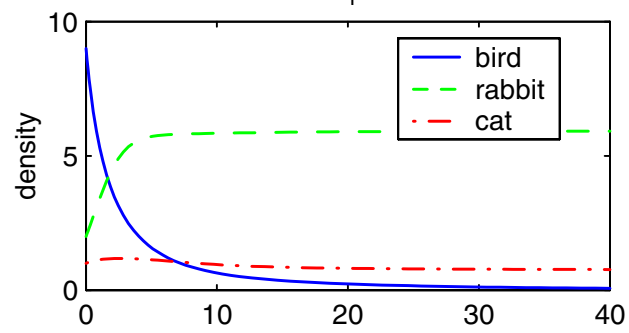

(d) $u_{\mathrm{r}}=1.2$

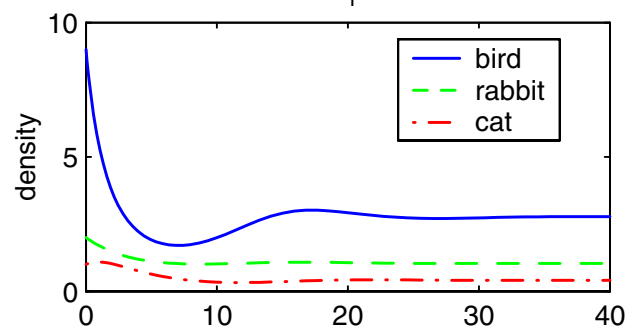

(f) bifurcation diagram for bird, cat

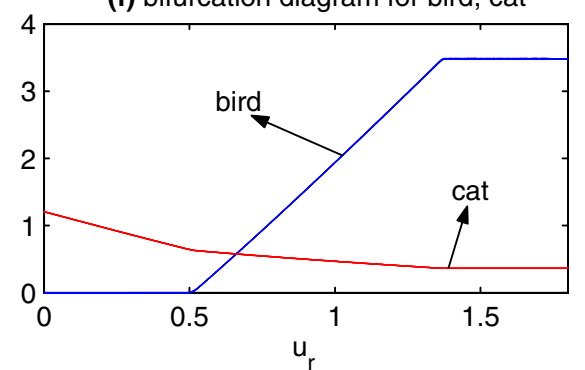

Fig. 2. (a,b) $E_{\mathrm{rc}}$ is attractor, where the birds is complete extinction, and the rabbits and the cats coexist. (c,d) The bird population recovers partly and $E_{\mathrm{brc}}$ become an attractor. (e) When the rabbits was eradicated, $E_{\mathrm{bc}}$ becomes an attractor. (f) Bifurcation diagram of the bird and the cat. Here $r_{1}=0.8, r_{2}=1.5, K_{1}=10, K_{2}=10, a_{1}=4, a_{2}=1$, $b_{1}=0.4, b_{2}=0.3, \alpha=0.2, \beta=0.05, \gamma=3, d=0.5, u_{\mathrm{c}}=0$.

First, we consider the bird's intrinsic growth rate $r_{1}$ and its carrying capacity $K_{1}$. By varying $r_{1}$ or $K_{1}$, one can determine the effect of these key parameters on the outcomes of the system. From Fig. 3(a), one can observe that increasing intrinsic growth rate of the birds can avoid the extinction induced by rabbits. Extensive numerical simulations show that $K_{1}$ fails to have similar effect.

We now investigate the effect of the parameters $\alpha$ and $\beta$, the predation efficiencies of the cat for the bird and the rabbit, on the dynamics of (4.1). $\alpha$ and $\beta$ can represent ability of anti-predation of the bird and the rabbit. For example, if $\alpha$ is bigger, then the cat has a higher capture rate for birds, which equivalently means that the bird has lower anti-predation ability. Recall that, in our original model construction, 
(a)

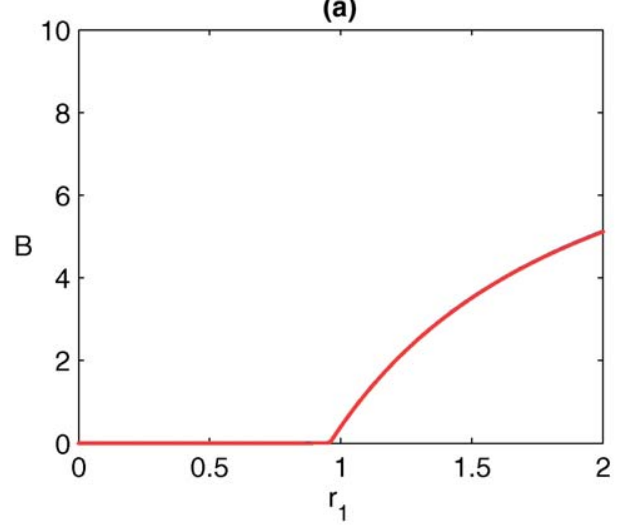

(c) $\beta=0.05$

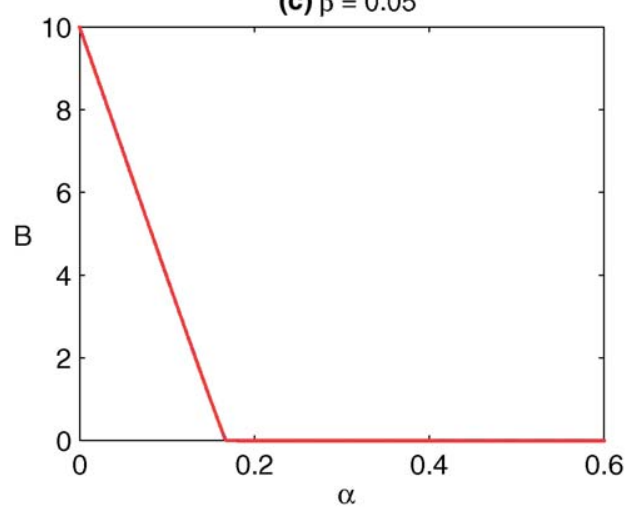

(b) bifurcation surface

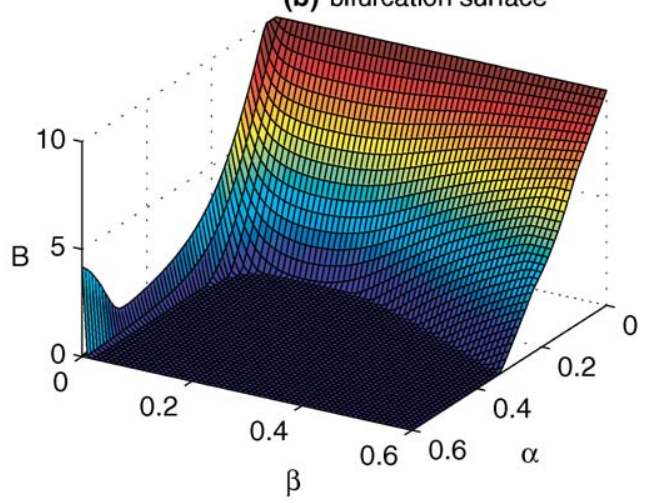

(d) $\alpha=0.2$

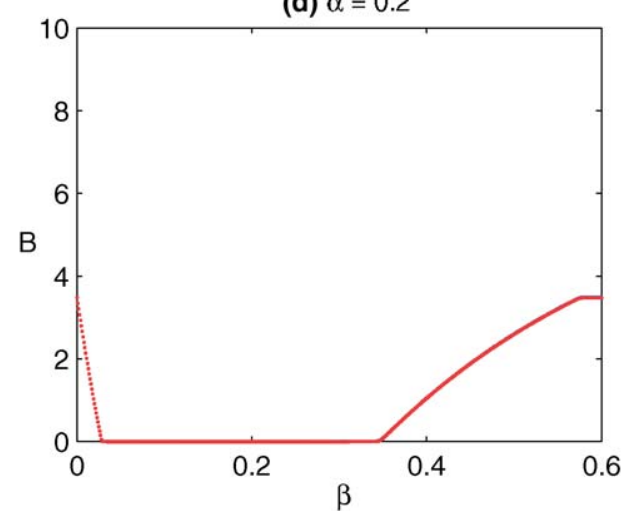

Fig. 3. The intrinsic growth rate of the bird and the anti-predation ability of the bird and the rabbit play key roles in killing the birds via hyperpredation. In (a), $r_{2}=1.5, K_{1}=10, K_{2}=10, a_{1}=4, a_{2}=1, b_{1}=0.4, b_{2}=0.3, \alpha=0.2$, $\beta=0.05, \gamma=3, d=0.5$; in (b), $r_{1}=0.8, r_{2}=1.5, K_{1}=10, K_{2}=10, d=0.5, \gamma=3, e_{1}=0.1, e_{2}=0.3, a_{1}=20 \alpha, a_{2}=20 \beta$, $b_{1}=e_{1} a_{1}, b_{2}=e_{2} a_{2}$.

$$
a_{1}=\frac{\alpha}{t_{\mathrm{chb}}}, \quad a_{2}=\frac{\beta}{t_{\mathrm{chr}}}, \quad b_{1}=\frac{e_{1} \alpha}{t_{\mathrm{chb}}}, \quad b_{2}=\frac{e_{2} \beta}{t_{\mathrm{chr}}} .
$$

From Fig. 3, one can observe that it is fatal for the bird to have a large $\alpha$. However, if the rabbits have a very weak or strong anti-predation trait, the birds may persist. This indicates that the hyperpredation may be the decisive factor of the rabbit induced bird extinction. To conclude, the ability of anti-predation of the bird and the rabbit play an important and decisive role in bird's persistence.

As mentioned by Courchamp [4], most indigenous prey which are able to cope with introduced predators will not be able to withstand the predation if an alien prey is also introduced, because of the hyperpredation process. The bird that cope well with the hyperpredation process must have a high intrinsic growth rate and adequate anti-predator defenses. However, in real ecosystems, especially in insular ecosystem, the indigenous prey generally has moderate intrinsic growth rate and 
poor anti-predator defenses. Therefore, hyperpredation represents a grave potential threat to the indigenous prey when both an alien predator and prey are introduced.

\section{Control of the rabbit or the cat}

As we have shown in previous section, the rabbit presents a grave threat to the indigenous prey when both the rabbit and the cat are introduced even when the direct effect is neglected. The harm caused by introduced species on oceanic islands is widely known, and control programs are carried out to restore or protect these ecosystems. As mentioned in the introduction, eradication of alien cat or rabbits populations is required in many cases since the alien cats can cause considerable damage to many managed and natural systems. However, the optimal strategy should be based on a careful study of the dynamics of the system. In this section, we will systematically explore optimal control strategies for protecting the bird. In the following discussion, we always assume that

$$
b_{2} \gamma>a_{2} \beta, \quad b_{1} \gamma>a_{1} \alpha, \quad a_{1}>r_{1} \gamma, \quad r_{2} a_{1}>r_{1} a_{2} .
$$

In order to facilitate our discussion below, we partition the parameter ranges in the $\left(u_{\mathrm{r}}, d+u_{\mathrm{c}}\right)$ plane according to the various dynamic scenarios of (2.3) (Fig. 4). Define

$$
\begin{aligned}
& \Delta_{b}=\left\{\left(u_{\mathrm{r}}, d+u_{\mathrm{c}}\right): u_{\mathrm{r}}>r_{2}, d+u_{\mathrm{c}}>\frac{b_{1} K_{1}}{K_{1} \alpha+1}\right\}, \\
& \Delta_{\mathrm{r}}=\left\{\left(u_{\mathrm{r}}, d+u_{\mathrm{c}}\right): \eta>\frac{r_{1}}{K_{2}}, 0 \leqslant u_{\mathrm{r}}<r_{2}\left(1-\frac{r_{1}}{\eta K_{2}}\right), d+u_{\mathrm{c}}>\delta_{1}\right\}, \\
& \Delta_{\mathrm{br}}=\left\{\left(u_{\mathrm{r}}, d+u_{\mathrm{c}}\right): 0 \leqslant \eta<\frac{r_{1}}{K_{2}}, 0 \leqslant u_{\mathrm{r}}<r_{2}, d+u_{\mathrm{c}}>\delta_{2}\right\}, \\
& \Delta_{\mathrm{br}}^{*}=\left\{\left(u_{\mathrm{r}}, d+u_{\mathrm{c}}\right): \eta>\frac{r_{1}}{K_{2}}, r_{2}\left(1-\frac{r_{1}}{\eta K_{2}}\right)<u_{\mathrm{r}}<r_{2}, d+u_{\mathrm{c}}>\delta_{2}\right\}, \\
& \Delta_{\mathrm{bc}}=\left\{\left(u_{\mathrm{r}}, d+u_{\mathrm{c}}\right): u_{\mathrm{r}}>\max \left\{r_{2}-\frac{a_{2}}{\gamma}, r_{2}-\frac{r_{1} a_{2}}{a_{1}}\right\}, 0<d+u_{\mathrm{c}}<\min \left\{\frac{b_{1} K_{1}}{K_{1} \alpha+1}, \delta_{3}\right\}\right\}, \\
& \Delta_{\mathrm{rc}}=\left\{\left(u_{\mathrm{r}}, d+u_{\mathrm{c}}\right): 0 \leqslant \eta<\frac{r_{1}}{K_{2}}, 0 \leqslant u_{\mathrm{r}}<r_{2}-\frac{r_{1} a_{2}}{a_{1}}, 0<d+u_{\mathrm{c}}<\min \left\{\delta_{1}, \delta_{4}\right\}\right\}, \\
& \Delta_{\mathrm{rc}}^{*}=\left\{\left(u_{\mathrm{r}}, d+u_{\mathrm{c}}\right): \frac{r_{1}}{K_{2}} \leqslant \eta<\frac{a_{1} r_{2}}{a_{2} K_{2}}, 0 \leqslant u_{\mathrm{r}}<r_{2}-\frac{r_{1} a_{2}}{a_{1}}, 0<d+u_{\mathrm{c}}<\min \left\{\delta_{1}, \delta_{4}\right\}\right\} \text { or } \\
& =\left\{\left(u_{\mathrm{r}}, d+u_{\mathrm{c}}\right): \eta \geqslant \frac{a_{1} r_{2}}{a_{2} K_{2}}, 0 \leqslant u_{\mathrm{r}}<r_{2}-\frac{r_{1} a_{2}}{a_{1}}, 0<d+u_{\mathrm{c}}<\delta_{1}\right\},
\end{aligned}
$$


(a)

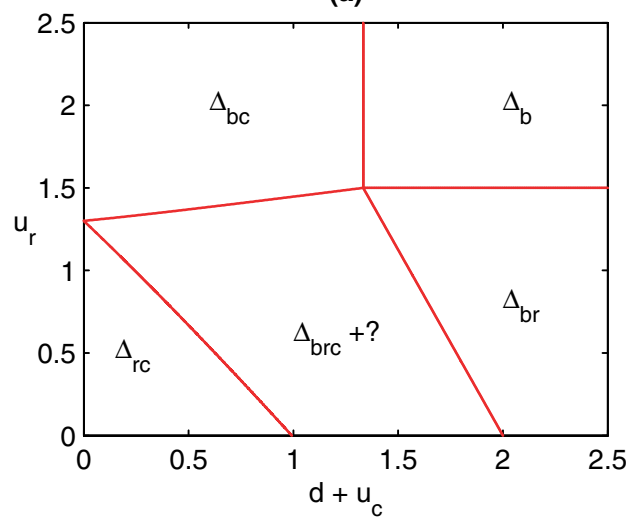

(b)

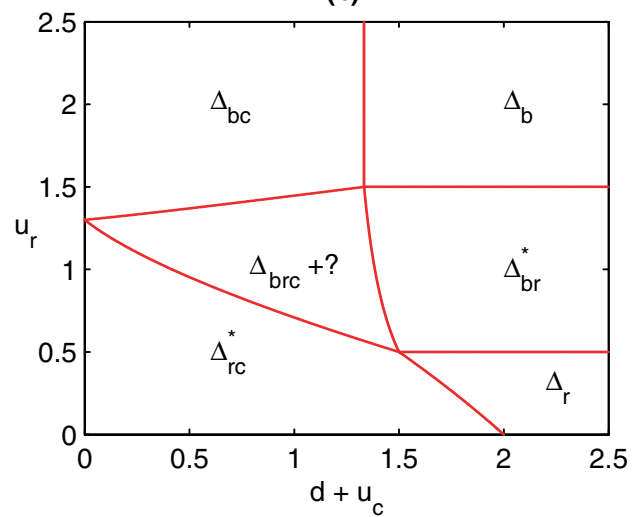

Fig. 4. Partition the parameter ranges in the $\left(u_{\mathrm{r}}, d+u_{\mathrm{c}}\right)$ plane according to the various dynamic scenarios of (2.3). Here $r_{1}=0.8, r_{2}=1.5, K_{1}=10, K_{2}=10, a_{1}=4, a_{2}=1, b_{1}=0.4, b_{2}=0.3, \alpha=0.2, \beta=0.05, \gamma=3, d=0.5$. (a) $\eta=0.02$. Here $0 \leqslant \eta<r_{1} / K_{2}$. (b) $\eta=0.12$. Here $\eta>r_{1} / K_{2}$.

where

$$
\begin{aligned}
\delta_{1} & =\frac{b_{2} K_{2}\left(r_{2}-u_{\mathrm{r}}\right)}{\beta K_{2}\left(r_{2}-u_{\mathrm{r}}\right)+r_{2}}, \\
\delta_{2} & =\frac{r_{1} r_{2} b_{1} K_{1}+K_{2}\left(r_{2}-u_{\mathrm{r}}\right)\left(r_{1} b_{2}-b_{1} \eta K_{1}\right)}{r_{1} r_{2}\left(1+\alpha K_{1}\right)+K_{2}\left(r_{2}-u_{\mathrm{r}}\right)\left(r_{1} \beta-\alpha \eta K_{1}\right)}, \\
\delta_{3} & =\frac{b_{1} K_{1}\left[r_{1} a_{2}-a_{1}\left(r_{2}-u_{\mathrm{r}}\right)\right]\left[a_{2}-\left(r_{2}-u_{\mathrm{r}}\right) \gamma\right]}{a_{2}\left[K_{1} \alpha\left(r_{1} a_{2}-a_{1}\left(r_{2}-u_{\mathrm{r}}\right)\right)+r_{1} a_{2}\right]}, \\
\delta_{4} & =\frac{b_{2} K_{2}\left[a_{1}\left(r_{2}-u_{\mathrm{r}}\right)-r_{1} a_{2}\right]\left[\eta K_{2} \gamma\left(r_{2}-u_{\mathrm{r}}\right)+\left(a_{1} r_{2}-a_{2} \eta K_{2}\right)-r_{1} r_{2} \gamma\right]}{\left(a_{1} r_{2}-a_{2} \eta K_{2}\right)\left[\left(a_{1} r_{2}-a_{2} \eta K_{2}\right)+K_{2} \beta\left(a_{1}\left(r_{2}-u_{\mathrm{r}}\right)-a_{2} r_{1}\right)\right]} .
\end{aligned}
$$

Fig. 4 illustrates the parameter ranges for various extinction scenarios and possible coexistence. It is clear that $\Delta_{\mathrm{r}}, \Delta_{\mathrm{rc}}, \Delta_{\mathrm{rc}}^{*}$ in Fig. 4 are dangerous regions for the birds since the bird component of the attractor of (2.3) is zero; that is, the extinction is the only possible fate of the birds. In $\Delta_{\mathrm{c}}, \Delta_{\mathrm{rc}}^{*}$, the hyperpredation process occurs.

\subsection{Rabbits have weak direct effect on birds}

In the following, we study the optimal control strategies for protecting the birds when $0 \leqslant \eta<r_{1} / K_{2}$, which means that the introduced prey (the rabbit) asserts no or weak direct effect on the indigenous prey. In Fig. 4(a), we depict the dynamic scenarios of (2.3) for this case in the $\left(u_{\mathrm{r}}, d+u_{\mathrm{c}}\right)$ plane. Theorem 3.2 tells us that $E_{\mathrm{r}}$ cannot be the attractor of (2.2) while Theorem 3.4 says that the bird is in an endangered situation (see Fig. 5(a), (g), and (h)). This is due to the fact that $E_{\mathrm{rc}}$ is an attractor of (2.2) when

$$
0<d<\frac{b_{2} K_{2}\left[a_{1} r_{2}-r_{1} a_{2}\right]\left[\eta K_{2} \gamma r_{2}+\left(a_{1} r_{2}-a_{2} \eta K_{2}\right)-r_{1} r_{2} \gamma\right]}{\left(a_{1} r_{2}-a_{2} \eta K_{2}\right)\left[\left(a_{1} r_{2}-a_{2} \eta K_{2}\right)+K_{2} \beta\left(a_{1} r_{2}-a_{2} r_{1}\right)\right]} .
$$


In order to protect the bird, we have several different choices: control the cats, control the rabbits, or control both the rabbits and the cats.

We investigate the strategy of controlling the cats first. If we increase the value of $u_{\mathrm{c}}$, then the system results in the coexistence of the birds, the cats and the rabbits, i.e., $E_{\mathrm{brc}}$ is the attractor of the system (see Fig. 5(d), (g), and (h)). If the control effort is sufficient enough, $d+u_{\mathrm{c}}>\frac{b_{1} K_{1}+b_{2} K_{2}}{1+\alpha K_{1}+\beta K_{2}}$, then the cats are eradicated and the birds and the rabbits coexist at $E_{\mathrm{br}}$ (see Fig. 5(e), (g), and (h)). After the eradication of the cats, sufficient control of the rabbits can help the bird increase toward its carrying capacity (see Fig. 5(f), (g), and (h)). We consider now the strategy of controlling the rabbit. After mild control of the rabbits, $E_{\mathrm{brc}}$ will become an attractor (see Fig. 5(b), (g), and (h)). When the rabbits are eradicated, $E_{\mathrm{bc}}$ is the attractor (see Fig. 5(c), (g), and (h)). With the rabbits eradicated, we can start the control of the cats. If we apply a control force strong enough, say, let $d+u_{\mathrm{c}}>\frac{b_{1} K_{1}}{1+\alpha K_{1}}$, then the bird population recovers completely (see Fig. 5(f), (g), and (h)). This shows, when the birds suffer weak to mild direct competition from the rabbits, both the control of the cats or the control of the rabbits can save the birds.

(a) $u_{r}=0 u_{c}=0(*)$

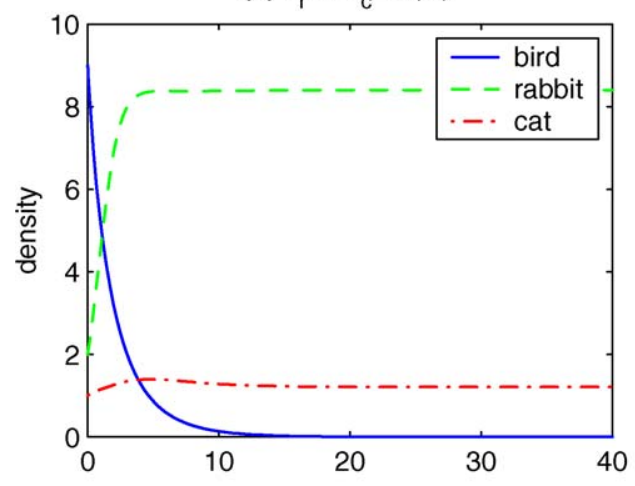

(b) $\mathrm{u}_{\mathrm{r}}=1 \mathrm{u}_{\mathrm{c}}=0(\oplus)$

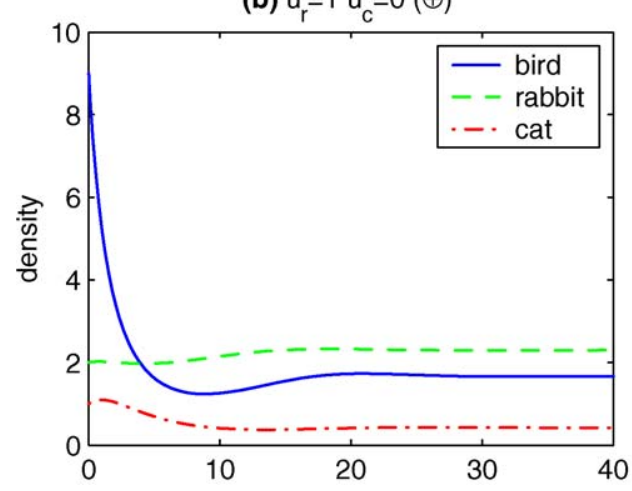

(c) $\mathrm{u}_{\mathrm{r}}=1.7 \mathrm{u}_{\mathrm{c}}=0(\oplus)$

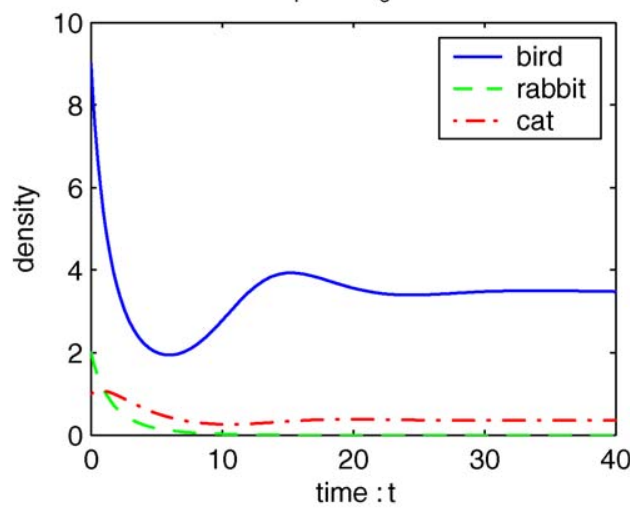

(d) $\mathrm{u}_{\mathrm{r}}=0 \mathrm{u}_{\mathrm{c}}=1(\otimes)$

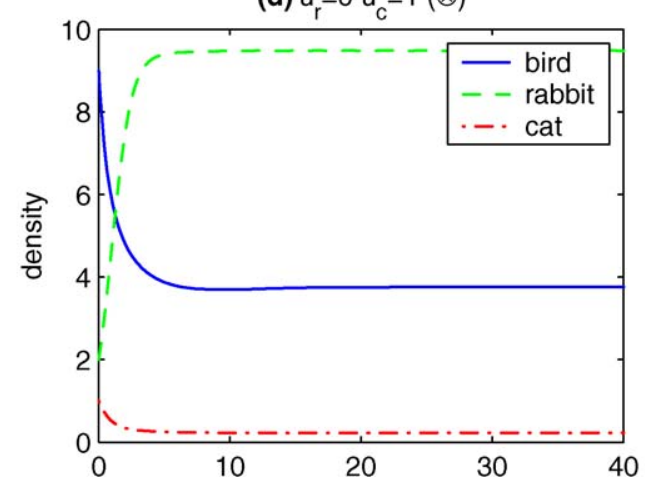

Fig. 5. Control strategies for protecting the birds when the rabbit has weak direct competition effect on the birds. (a) Without any control; (b,c) control the rabbit only; (d,e) control the cat only; (f) control both the rabbit and the cat; (g) control strategies; (h) bifurcation surface: bird versus $u_{\mathrm{r}}$ and $u_{\mathrm{c}}$. Here $r_{1}=0.8, r_{2}=1.5, K_{1}=10, K_{2}=10, a_{1}=4, a_{2}=1$, $b_{1}=0.4, b_{2}=0.3, \alpha=0.2, \beta=0.05, \gamma=3, \eta=0.02, d=0.5$. 
(e) $u_{r}=0 u_{c}=1.7(\otimes)$

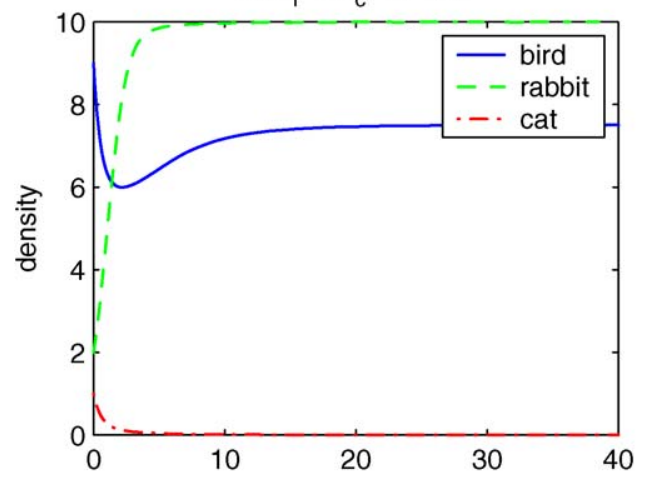

(g) control strategies

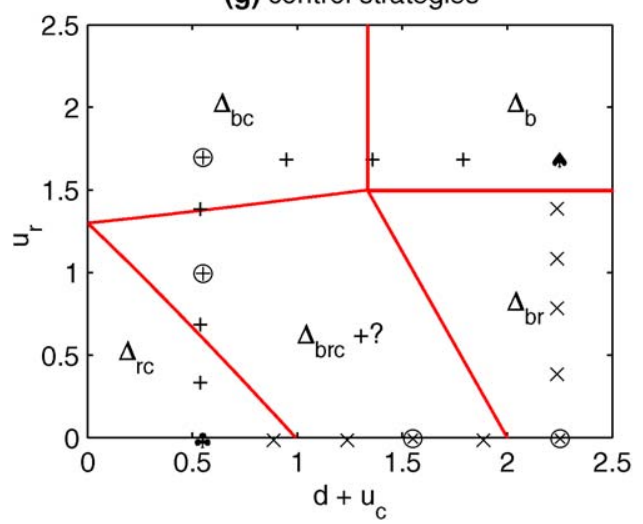

(f) $u_{r}=1.7 u_{c}=1.7(\wedge)$

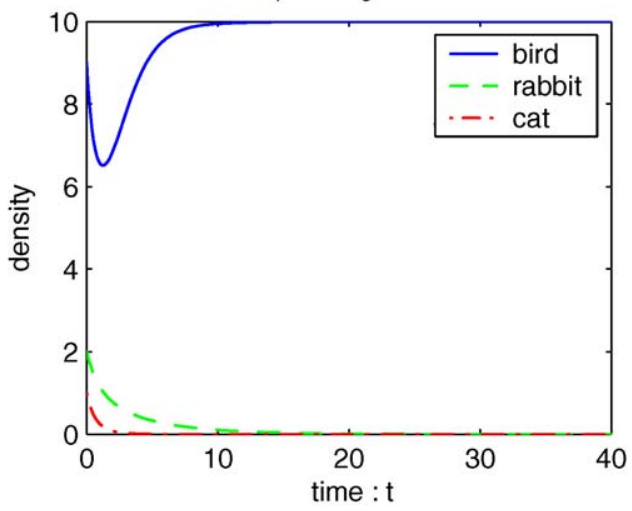

(h) bifurcation surface

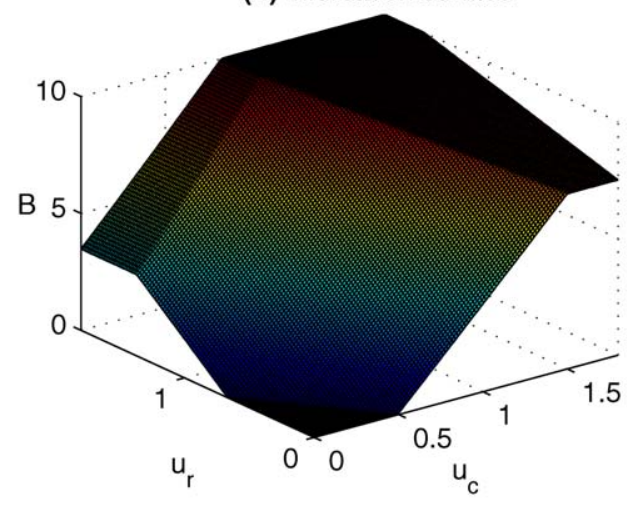

Fig. 5 (continued)

\subsection{Rabbits have strong direct effect on birds}

Now we continue with the case $\eta>r_{1} / K_{2}$, where the birds suffer strong direct competition effect from the rabbits. In Fig. 4(b), we depict the dynamic scenarios of (2.3) for this case in the $\left(u_{\mathrm{r}},-\right.$ $d+u_{\mathrm{c}}$ ) plane. In this case, from Theorems 3.2 and 3.4, we know that both $E_{\mathrm{r}}$ and $E_{\mathrm{rc}}$ are possible attractors of (2.2). If

$$
b_{2} \gamma>a_{2} \beta, \quad r_{2} a_{1}>r_{1} a_{2}, \quad 0<d<\frac{b_{2} K_{2}}{1+\beta K_{2}},
$$

then $E_{\mathrm{rc}}$ is the attractor of (2.2) and hence the birds are endangered (see Fig. 6(a), (g), and (h)).

We may start by controlling the cat only $\left(u_{\mathrm{r}} \equiv 0\right)$. For small or moderate control, $E_{\mathrm{rc}}$ is the attractor of (2.3). When $d+u_{\mathrm{c}}>b_{2} K_{2} /\left(1+\beta K_{2}\right), E_{\mathrm{r}}$ becomes the attractor of $(2.3)$ and the birds are in great danger. From Fig. $6(\mathrm{~d}),(\mathrm{e}),(\mathrm{g})$, and (h), one can see that, without the control of the rabbit, any control of the cats is useless for saving the birds. So, in order to protect the birds, we have to control the rabbits first. With increasing the control of the rabbit, the attractor of (2.3) is 
$E_{\text {rc }}$ (insufficient control; see Fig. 6(g) and (h)), $E_{\text {brc }}$ (moderate control; see Fig. 6(b), (g), and (h)) and $E_{\mathrm{bc}}$ (sufficient control; see Fig. 6(c), (g), and (h)). In order to fully resurrect the birds, one must also control the cats. With sufficient control of the cats, the bird population can reach its carrying capacity (see Fig. 6(f), (g), and (h)).

\section{Conclusions}

In this paper, we have developed a plausible model (2.3) describing the dynamic interactions among an indigenous prey (bird), an introduced prey (rabbit) and a common introduced predator (cat) in an island. Our model overcomes several model formulation problems arising in system (1.1) in [4]. We show that (2.3) can admit rich and realistic dynamics and hence provides insights for the design of control programs.

With the model (4.1), we confirm that the apparent competition or the hyperpredation process does occur in the bird-rabbit-cat system; that is, even when the rabbit does not have any direct
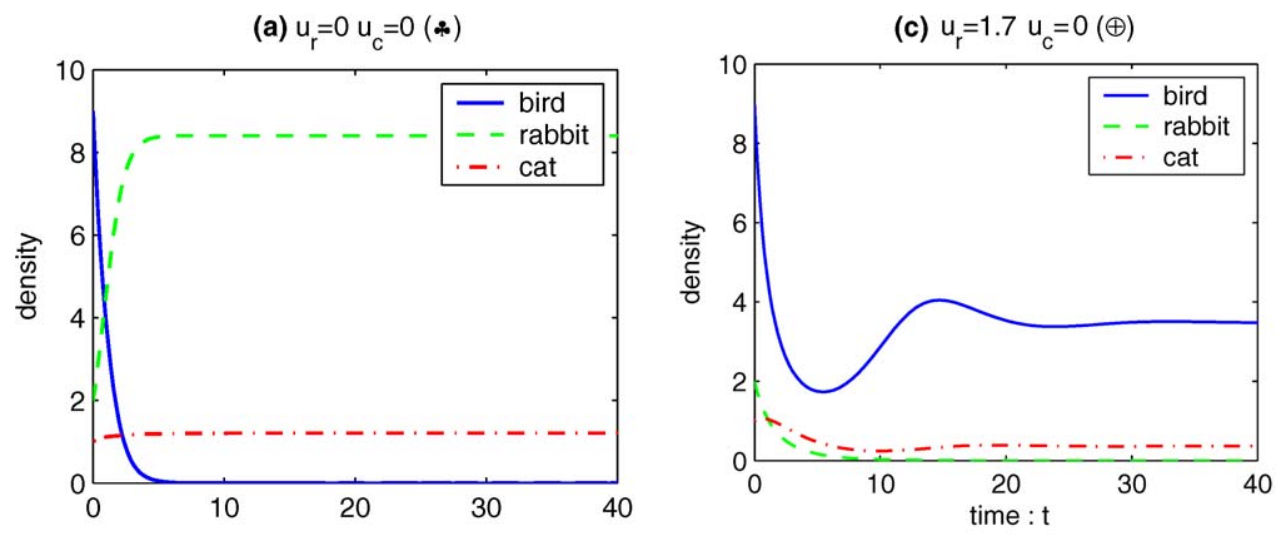

(b) $\mathrm{u}_{\mathrm{r}}=1 \mathrm{u}_{\mathrm{c}}=0(\oplus)$
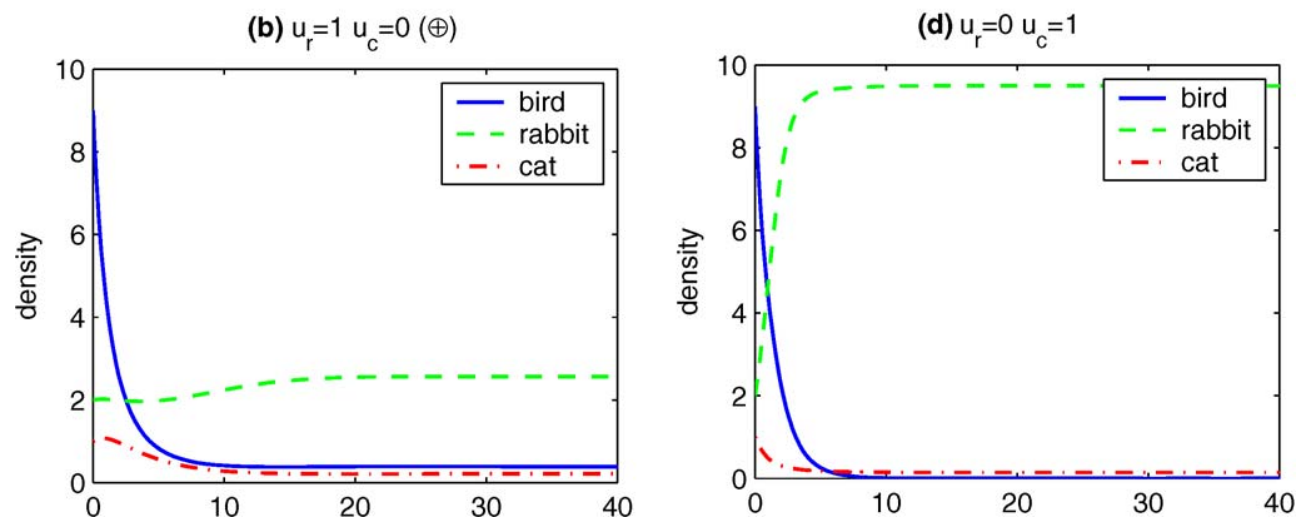

Fig. 6. Control strategies for protecting the birds when the rabbit has strong direct competition effect on the birds. (a) Without any control; (b,c) control the rabbit only; (d,e) control the cat only; (f) control both the rabbit and the cat; (g) control strategies; (h) bifurcation surface: bird versus $u_{\mathrm{r}}$ and $u_{\mathrm{c}}$. Here $r_{1}=0.8, r_{2}=1.5, K_{1}=10, K_{2}=10, a_{1}=4, a_{2}=1$, $b_{1}=0.4, b_{2}=0.3, \alpha=0.2, \beta=0.05, \gamma=3, \eta=0.12, d=0.5$. 

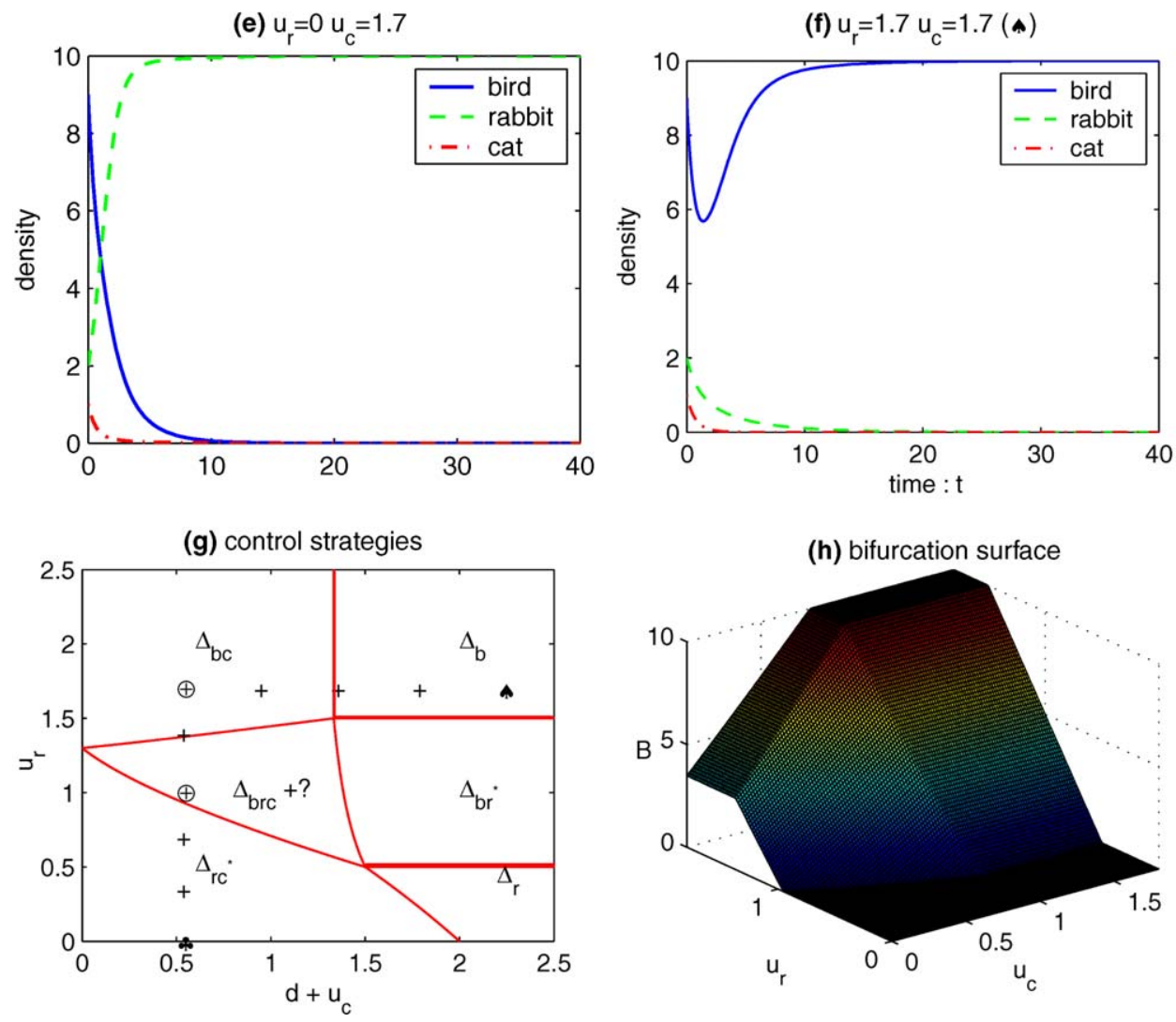

Fig. 6 (continued)

negative effect on the bird, the rabbit can greatly increase the growth of the cat and indirectly asserts heavy predation on the bird through the cat and therefore leads to the extinction of the birds. With (4.1), we can further quantify the timing of the hyperpredation process and its detrimental effect on the indigenous prey. It shows that the basic conditions required for an endemic prey to survive the hyperpredation process include high intrinsic growth rate and adequate anti-predation ability. However, if the birds have very poor anti-predation behavior and at the same time, the rabbits have a strong anti-predation, the birds my still persist.

Based on (2.3), our study of the control strategies shows that the fate of the prey sensitively depends on both the alien prey control level and the alien (common) predator control level. The presence of the introduced prey can allow an increased predator population, which can lead to the extinction of the endemic prey, and increase the difficulty of the predator control. With model (2.3) and numerical analysis, we show that the control of the introduced prey can facilitate the control of the introduced predator. Moreover, predator control may fail to protect the endemic prey if the control of the introduced prey is not controlled simultaneously when the alien prey asserts strong direct competition effect on the endemic prey. Therefore, control of both introduced prey and predator represents the best strategy to protect the bird. 
In real applications, if the ecosystem on some island can be well described by the prey-alien prey-alien predator trophic web and the indigenous prey species is endangered, we shall be wary about any intuitive control strategy aimed to protect the endemic prey species. To obtain a sound control strategy, we should first determine the status of the ecosystem, i.e., determine the parameters in (2.2), then study the qualitative dynamics of the system. It is a good idea to compute some diagrams similar to Fig. 4 and determine the positions of the system. Sound control strategies based on the above discussion and other policy restrictions will likely emerge.

As discussed in the end of Section 3, the instability of $E_{0}$ is mathematical and somewhat troublesome in this system since extinction in this closed system is biologically stable. This discrepancy can be explained by several ways. One natural explanation is that animals are discrete and can be regarded as zero when the densities become very small. This can happen even though the equilibrium at the origin $E_{0}$ is unstable. Another popular explanation is that the stochastic forces are ubiquitous and when they are accounted in mathematical models, then the instability of $E_{0}$ may be lost. A more recent and intriguing explanation is that in the limiting scenarios when searching time is very small (like in a small island, or fragmented small patches), our functional responses can be approximated by the so-called ratio-dependent functional responses [10,14,15]. For such functionals, the origin can indeed be an attractor.

A yet to be addressed mathematical question on (2.3) is under what conditions, all three species coexist. This is the so-called persistence question. In this paper, we do not study this interesting topic since persistence issue is not directly relevant to biological control applications and the hyperpredation process. An even more intriguing mathematical question is whether (2.3) is capable of generating cyclic dynamics and/or chaotic dynamics. Our intensive computational efforts so far failed to detect that.

\section{Acknowledgments}

We would like to thank Professor Donald L. DeAngelis and the referees for their careful readings of the manuscript and many thoughtful suggestions that lead to a much improved exposition of this manuscript.

\section{References}

[1] P.A. Abrams, R.D. Holt, J.D. Roth, Apparent competition or apparent mutualism? Shared predation when populations cycle, Ecology 79 (1998) 201.

[2] J.R. Beddington, Mutual interference between parasites or predators and its effect on searching efficiency, J. Anim. Ecol. 44 (1975) 331.

[3] R.S. Cantrell, C. Cosner, On the dynamics of predator-prey models with the Beddington-DeAngelis functional response, J. Math. Anal. Appl. 257 (2001) 206.

[4] F. Courchamp, M. Langlais, G. Sugihara, Control of rabbits to protect island birds from cat predation, Biol. Conserv. 89 (1999) 219.

[5] F. Courchamp, M. Langlais, G. Sugihara, Cats protecting birds: modelling the mesopredato release effect, J. Anim. Ecol. 68 (1999) 282.

[6] G. Courchamp, M. Langlais, G. Sugihara, Rabbits killing birds: modelling the hyperpredation process, J. Anim. Ecol. 69 (2000) 154. 
[7] D.L. DeAngelis, R.A. Goldstein, R.V. O’Neill, A model for trophic interaction, Ecology 56 (1975) 881.

[8] M. Fan, Y. Kuang, Z.L. Feng, Cats protecting birds revisited, Bull. Math. Biol. 67 (2005) 1081.

[9] R.D. Holt, Predation, apparent competition, and the structure of prey communities, Theor. Popul. Biol. 12 (1977) 275.

[10] S.-B. Hsu, T.-W. Hwang, Y. Kuang, A ratio-dependent food chain model and its applications to biological control, Math. Biosci. 181 (2003) 55.

[11] T.-W. Hwang, Global analysis of the predator-prey system with Beddington-DeAngelis functional response, J. Math. Anal. Appl. 281 (2003) 395.

[12] T.-W. Hwang, Uniqueness of limit cycles of the predator-prey system with Beddington-DeAngelis functional response, J. Math. Anal. Appl. 290 (2004) 113.

[13] C. Jost, S.P. Ellner, Testing for predator dependence in predator-prey dynamics: a non-parametric approach, Proc. R. Soc. Lond. Ser. B 267 (2000) 1611.

[14] Y. Kuang, Rich dynamics of Gause-type ratio-dependent predator-prey system, Fields Inst. Commun. 21 (1999) 325.

[15] Y. Kuang, E. Beretta, Global qualitative analysis of a ratio-dependent predator-prey system, J. Math. Biol. 36 (1998) 389.

[16] Y. Kuang, F. Fagan, I. Loladze, Biodiversity, habitat area, resource growth rate and interference competition, Bull. Math. Biol. 65 (2003) 497.

[17] F.I. Norman, Long term effects of rabbit reduction on Rabbit Island Wilson's Promontory Victoria Australia, Victorian Natrualist (South Yarra) 105 (1988) 136.

[18] S.G. North, D.J. Bullock, M.E. Dulloo, Changes in the vegetation and reptile populations on Round Island, Mauritius, following eradication of rabbits, Biol. Conser. 67 (1994) 21.

[19] G.W. Roemer, C.J. Donlan, F. Courchamp, Golden eagles, feral pigs, and insular carnivores: how exotic species turn native predators into prey, Proc. Natl. Acad. Sci. USA 22 (2002) 791. 